A CTO is a flexible tool in the early stages of actual use in the community. Gathering the quality outcomes is still at an infancy stage and a balance is required to protect service user rights while keeping administration protocols to its minimum.

*Masood Malik Consultant Psychiatrist, North Wales NHS Trust, Pwll Glas Resource Centre, Pwl Glass Road, Mold, Flintshire CH7 1RA a Nafisa Hussein Associate Specialist, Cheshire and Wirral Partnership NHS FoundationTrust

doi: 10.1192/pb.33.11.437a

\section{Internet and doctors' security - how exposed are you?}

We have read with some concern on the College website the highlighting of personal information on electoral registers, its availability to the general public and the consequent security implications for mental health professionals. Members of the public have access to the edited electoral roll containing information such as home address, but as advised on the website, we can choose to opt out of this particular register.

Other than the electoral roll, doctors should also exercise caution as to how much personal information they divulge when using the internet and be aware as to how this information is shared and how accessible it is to others. The use of social networking sites has increased in recent times and search engines have become more advanced in seeking out information. When using them, extra security precautions should be taken.

Clearly, the more information we reveal and others divulge about us, the more information someone can use to build a comprehensive profile about us. This led us to conduct a qualitative study involving 18 mental health professionals from different disciplines of the same team. Each member was asked to conduct a basic internet-based search engine query about another team member. From the search results we listed what information could be sought about that person. None of the information we collected involved monetary payment

The results showed personal information about team members and consequent security risks. For a profession that is focused on risk assessment, we are not as cautious in our private lives. We were able to ascertain information about family details including children, personal photographs, personal telephone numbers and home addresses, dates of birth, workplace information and in one case, particular information where and when that person was going to be on a particular day. We were even able to discover which books a member of the team had bought online and which ones they wished to purchase.

We believe this is all potentially sensitive information which could be used with disastrous consequences such as identity fraud, harassment, stalking or worse, acts of violence. We are not just making targets of ourselves but also of friends and family. As workers in a discipline where risk is continuously considered, we should exercise caution as to the information we share in our private lives.

We do not wish to act as killjoys, advising not to use social networking sites, but we advocate using extra steps to limit information availability and to seek advice on how to limit breaches of security. This advice applies not only to psychiatrists but to all healthcare professionals

*Asad Raffi Specialty Registrar Year 6, Cheshire and Wirral Partnership NHS Trust, Churton Unit, Bowmere Hospital, Chester CH2 1BQ, email: asad.raffi@cwp.nhs.uk, Aisha Malik Genera Practice Registrar, North Lancashire Primary Care Trust, Jahangir Mahmoo Specialty Registrar Year 6, Mersey Care NHS Trust

doi: $10.1192 / \mathrm{pb} .33 .11 .438$

\section{The 'special' ones: survey of Laughlin Prize winners}

It is with a mixture of admiration and slight envy that I look upon those who triumph as best of the best in tough competitive examinations. Surely, to emerge on top they have to be 'special'. The Laughlin Prize, established in 1979, is awarded after the spring and autumn MRCPsych Part II examinations to the candidate obtaining high marks and the best recommendation from the examiners.

Given the above, I wished to find out more about the winners, their preparation for examination and whether winning the prize influenced their later career choice. I also hoped that the information obtained from this exercise would inspire trainees preparing for MRCPsych examinations. So I set out to conduct a postal questionnaire survey of all winners and contacted the College for a list of names. However, for confidentiality and data protection reasons, I was only given the names of those who won the prize since 2000 .

I piloted the questionnaire on one winner; the final version was designed to gather some demographic information and also consisted of open-ended questions such as 'Did winning the prize influence your career in any way?', 'Did winning the prize change others' perceptions of you?' and 'Was you preparation for the examination any different?' I sent out the questionnaire to the 12 winners identified and received seven replies. Below is a brief summary of the results.

Mean age 33 years (range 27-40); gender: male 5, female 2; ethnicity: White 5, Indian 2; country of medical training: UK 4, India 2, Australia 1; current subspecialty: general adult psychiatry 3 , child psychiatry 1 , old age psychiatry 1 , liaison psychiatry 1 , forensic psychiatry 1 ; self-rating of the importance of the prize in terms of career path (rated on a scale of 1-10): 5.7 mean, range $2-8$.

Five individuals reported that winning the prize did not influence their career in any significant way, although four of them felt that maybe it did help them get jobs and increased their overall confidence. Two respondents felt that it did influence their career choice (one towards a clinical career and one towards an academic post). Only one person said they probably worked harder than their peers, whereas others said their exam preparation was no different. Interestingly, two respondents said: 'I tried to enjoy the clinical examination', and 'Before the exam I realised that I had become too goal-orientated and that I needed to start enjoying being with patients'.

In summary, given the limited number of responses, no valid conclusions can be drawn. The predictive validity of the Laughlin Prize could also not be assessed, as the sample only comprised winners since 2000. Although there were more males (5/7) and White individuals (5/7) in this cohort, given the sample size, attempting to explore reasons for that would be merely speculative. Majority rated the prize highly in terms of its importance in their career path, although only two respondents actually felt it influenced their subsequent subspecialty or academic/clinical career choice. It is to be noted that those who said the prize did not influence their career choice had already made clear plans for their future, even before taking the examination. Although most respondents (6/7) said their preparation for the examination was no different to their peers, it is worth noting that two reported trying to 'enjoy the experience'. This might be an important message for trainees in that rather than trying to see the exam as an 'artificial' and stressful experience, maybe they should anticipate it as more of an enjoyable experience, no different to their daily doctor-patient interactions.

Having read through the responses (and as was pointed out by one of the winners), it was felt that the winners of the Laughlin Prize were inadequately acknowledged for their achievement. Surely to be the best among a cohort of 500 -odd doctors is no mean feat and is indeed, in my view, something special. If future trainees are to be inspired to work harder, maybe the College (Exams Department) could do more to 
acknowledge these 'special ones': maybe by publishing their names on the College website or interviewing them for the Psychiatric Bulletin. Someone once said that 'The most intelligent and the most competitive are the most reluctant to acknowledge their peers'. Is this the case, and if so, should it not change?

If any Laughlin Prize winner over the past 20 years wishes to contact me, I would like to repeat the survey.

\section{Declaration of interest}

S.G. did not win the Laughlin Prize and the views expressed here are solely his own.

\section{Acknowledgements \\ I thank all respondents.}

Sanju George Consultant in Addiction Psychiatry, Birmingham and Solihull Mental Health NHS FoundationTrust, The Bridge Clinic, Larch Croft ChelmsleyWood, Birmingham B37 7UR, email: sanju.george@bsmhft.nhs.uk

doi: $10.1192 / p b .33 .11 .438 a$

\section{Is this not discrimination?}

During one of the recent ward rounds, we decided to discharge a patient who had a diagnosis of schizoaffective disorder. He had been on the ward for about 2 months as a voluntary patient and he was treated with antipsychotic medication. We advised him that he should continue the medication on a daily basis after the discharge in order to prevent future relapse. He said that he had to pay for prescriptions and therefore expressed his difficulty in taking the antipsychotic medication on a longterm basis.

Research by Rethink (unpublished; personal communication, 2009) shows that $26 \%$ of service users currently pay for prescriptions $(56 \%$ of those who have been in paid employment over the past 12 months); $38 \%$ of people with severe mental illnesses like schizophrenia have to choose between paying household bills and prescription charges (www. prescriptionpromise.org). This group of patients will now have to pay even more due to the recent increase in prescription charges, which many already cannot afford. This grossly unjust situation can result in people not getting their medicines and as a result having a relapse of their mental illness.

Patients with chronic conditions such as hypothyroidism and diabetes mellitus are receiving free medication irrespective of their job or financial background (www. nhsbsa.nhs.uk/1126. aspx). Prescription charges have also been abolished recently for people with cancer, and the Parkinson's Disease Society has now urged the government to end prescription charges in England for people with
Parkinson's disease. Why not those with enduring mental health difficulties? Although a number of patients with chronic mental health problems do get free medication owing to their poor financial status, there are patients who may have some money in the bank or other income. It is not fair to presume that all psychiatric patients do not have any money and all of them are not capable of generating an income or even not entitled to inherit wealth.

The ethos for providing free medication in chronic conditions such as hypothyroidism should be valid and applicable to those with long-term mental health difficulties.

The College, along with the voluntary sector, should actively campaign to put pressure on the government to abolish prescription charges for people with longterm mental illness

*Manoj K. George Specialty Registrar in Psychiatry, North Essex Partnership NHS Foundation Trust-Mental Health, The Derwent Centre, Princess Alexandra Hospital, Hamstel Road, Harlow CM20 1QX, email: Manoj.George@nhs.net, Renju Joseph Clinical Director, Consultant Psychiatrist, Dudley and Walsall Mental Health Partnership NHS Trust, Bushey Fields Hospital, Dudley

doi: $10.1192 / p b .33 .11 .439$

\section{HoNOSty: does HoNOS provide a good enough measure of outcome?}

Sugarman et al highlight an important aspect of mental healthcare in their paper which analyses the use of Health of the Nation Outcome Scales (HoNOS) in assessing change among psychiatric patients. ${ }^{1}$ Although they demonstrate that it is possible to measure outcomes using a suitable instrument, their data also reveal the limited utility of such a 'blunt instrument' and they provide an honest account of its shortcomings, recommending that it should not be used alone. Indeed, the tiny degrees of change indicated in their results have little meaning for real-life clinical practice.

Historically, measuring clinical outcomes in psychiatry was discarded as anathema ${ }^{2}$ despite the availability of a variety of simple, quick and meaningful scales in many major conditions: the Beck Depression Inventory and the Hamilton scales for depression and anxiety being examples. It is essential that clinicians engage with the use of outcome measures, especially in light of the drive towards the use of outcomes in commissioning services, payment by results and the public availability of information about health providers that is now being published online.

A further demand, shaped by the Next Stage Review ${ }^{3}$ and various National Institute for Health and Clinical Excellence guidelines, is for measures to underpin the shared care of patients between primary and secondary care services. The concept of remission is of use here and has been well established in connection with depression ${ }^{4}$ but with few other mental disorders. Recent work on schizophrenia has provided a well-argued case for remission criteria to support shared care of patients with this disorder. The instrument, derived from the Positive and Negative Syndrome Scale, takes 10 minutes to administer and provides a simple, meaningful result for clinicians, patients and carers. Remission criteria have the additional advantage over HoNOS of being specific to the challenges experienced by patients with these disorders, both as an objective snapshot of the state of an individual's illness and as a marker of their long-term stability. ${ }^{5}$

The use of dynamic markers such as remission scores to measure progress is actively encouraged in the World Health Organization's Mental Health Gap Action Programme. ${ }^{6}$ If new models of healthcare are to be evaluated properly and the engagement of stakeholders facilitated, validated assessment of patient change will need to be performed to ensure both continued success and continued funding.

1 Sugarman P, Walker L, Dickens G. Managing outcome performance in mental health using HoNOS: experience at St Andrew's Healthcare. Psychiatr Bull 2009; 33: 285-8.

2 Jacques J. Payment by results and mental health services. Psychiatr Bull 2008; 32: 361-3.

3 Department of Health. NHS Next Stage Review Final Report. TSO (The Stationery Office), 2008.

4 Frank E, Prien RF, Jarret RB, Keller MB, Kupfer DJ, Lavori PW, et al. Conceptualisation and rationale for consensus definitions of terms in major depressive disorder: remission, recovery, relapse, and recurrence. Arch Gen Psychiatry 1991; 50: 407-8.

5 Yeomans D, Taylor M, CurrieA, Whale R, Ford K, Fear $C$, et al. How long have you been well? Remission as an outcome in schizophrenia. Advan PsychiatrTreat 2009; in press.

6 World Health Organization. mhGAP: Mental Health Gap Action Programme: Scaling up Care for Mental, Neurological and Substance Use Disorders. WHO Press, 2008.

*Benjamin J. Sieniewicz Foundation 2 Doctor, Gloucestershire Foundation Trust, Wotton Lawn Hospital, Horton Road, Gloucester GL1 3WL, email: benjamin.sieniewicz@glos.nhs.uk, Chris Fear Consultant Psychiatrist, 2gether NHS Foundation Trust

doi: 10.1192/pb.33.11.439a

\section{Involvement of a young service user in the appointment of a doctor}

The importance of involving service users in all aspects of their care has been 